\title{
Guideline update for the performance of fusion procedures for degenerative disease of the lumbar spine. Part 9: Lumbar fusion for stenosis with spondylolisthesis
}

\author{
Daniel K. Resnick, M.D., ${ }^{1}$ William C. Watters III, M.D., ${ }^{2}$ Alok Sharan, M.D. ${ }^{3}$ \\ Praveen V. Mummaneni, M.D., ${ }^{4}$ Andrew T. Dalley, M.D.,${ }^{5}$ Jeffrey C. Wang, M.D., 6 \\ Tanvir F. Choudhri, M.D. ${ }^{7}$ Jason Eck, D.O., M.S., ${ }^{8}$ Zoher Ghogawala, M.D., ${ }^{9}$ \\ Michael W. Groff, M.D., ${ }^{10}$ Sanjay S. Dhall, M.D., ${ }^{4}$ and Michael G. Kaiser, M.D. ${ }^{11}$ \\ ${ }^{1}$ Department of Neurosurgery, University of Wisconsin, Madison, Wisconsin; ${ }^{2}$ Bone and Joint Clinic of \\ Houston, Houston, Texas, ${ }^{3}$ Department of Orthopaedic Surgery, Montefiore Medical Center, Albert Einstein \\ College of Medicine, Bronx, New York; ${ }^{4}$ Department of Neurological Surgery, University of California, San \\ Francisco, California; ${ }^{5}$ Department of Neurosurgery, University of Utah, Salt Lake City, Utah; ${ }^{6}$ Department \\ of Orthopaedic Surgery, Keck School of Medicine, University of Southern California, Los Angeles, \\ California; ${ }^{7}$ Department of Neurosurgery, Icahn School of Medicine at Mount Sinai, New York, New York; \\ ${ }^{8}$ Center for Sports Medicine and Orthopaedics, Chattanooga, Tennessee; ${ }^{9}$ Alan and Jacqueline Stuart Spine \\ Research Center, Department of Neurosurgery, Lahey Clinic, Burlington, and Tufts University School of \\ Medicine, Boston, Massachusetts; ${ }^{10}$ Department of Neurosurgery, Brigham and Women's Hospital, Boston, \\ Massachusetts; and ${ }^{11}$ Department of Neurosurgery, Columbia University, New York, New York
}

\begin{abstract}
Patients presenting with stenosis associated with a spondylolisthesis will often describe signs and symptoms consistent with neurogenic claudication, radiculopathy, and/or low-back pain. The primary objective of surgery, when deemed appropriate, is to decompress the neural elements. As a result of the decompression, the inherent instability associated with the spondylolisthesis may progress and lead to further misalignment that results in pain or recurrence of neurological complaints. Under these circumstances, lumbar fusion is considered appropriate to stabilize the spine and prevent delayed deterioration. Since publication of the original guidelines there have been a significant number of studies published that continue to support the utility of lumbar fusion for patients presenting with stenosis and spondylolisthesis. Several recently published trials, including the Spine Patient Outcomes Research Trial, are among the largest prospective randomized investigations of this issue. Despite limitations of study design or execution, these trials have consistently demonstrated superior outcomes when patients undergo surgery, with the majority undergoing some type of lumbar fusion procedure. There is insufficient evidence, however, to recommend a standard approach to achieve a solid arthrodesis. When formulating the most appropriate surgical strategy, it is recommended that an individualized approach be adopted, one that takes into consideration the patient's unique anatomical constraints and desires, as well as surgeon's experience.
\end{abstract}

(http://thejns.org/doi/abs/10.3171/2014.4.SPINE14274)

KEY WORDS • fusion • lumbar spine • spondylolisthesis
practice guidelines

\section{Recommendations}

There is no evidence that conflicts with the previous recommendations formulated from the first iteration of the Lumbar Fusion Guidelines.

\footnotetext{
Abbreviations used in this paper: ODI = Oswestry Disability Index; PLF = posterolateral lumbar fusion; PLIF = posterior lumbar interbody fusion; SPORT $=$ Spine Patient Outcomes Research Trial; TLIF = transforaminal lumbar interbody fusion; VAS = visual analog scale.
}

Grade B

Surgical decompression and fusion is recommended as an effective treatment alternative for symptomatic stenosis associated with a degenerative spondylolisthesis in patients who desire surgical treatment.

Although there is insufficient evidence to recommend a standard fusion technique, the patient's anatomy, desires, and concerns as well as surgeon experience should all be factored into the decision-making process when determining the optimal strategy for an individual patient to maximize fusion potential while minimizing risk of complications. 
Part 9: Lumbar fusion for stenosis with spondylolisthesis

\section{Rationale}

Patients presenting with clinically relevant stenosis associated with a spondylolisthesis may report signs and symptoms consistent with neurogenic claudication, radiculopathy, and/or low-back pain. A decompressive procedure is often required to alleviate the symptoms associated with the neurological compression syndrome; however, decompression alone can result in progression of the vertebral misalignment. In the original version of the Lumbar Fusion Guidelines, incorporating a posterolateral lumbar fusion (PLF) as an adjunct to a lumbar decompression was considered an appropriate treatment alternative to prevent deformity progression and improve patient outcomes. Supplementation of the PLF with pedicle screw stabilization was considered an appropriate option in the presence of a kyphosis or if instability was suspected. ${ }^{26}$ The purpose of the current Guideline Update was to examine the current literature investigating the role of surgical intervention for patients with symptomatic stenosis associated with spondylolisthesis and focus on the utility of lumbar fusion in this patient population.

\section{Literature Search}

Several well-publicized randomized controlled clinical trials have been published since the last systematic review published in 2005. ${ }^{25}$ Accordingly, the literature search strategy was designed to reflect the existence of potentially high-quality evidence. The National Library of Medicine and the Cochrane Library were searched for articles published between July 2003 and December 2011, using an electronic literature search engine (PubMed and the Cochrane Search Engine, respectively) with the following subject headings: (((“Lumbosacral Region" [MeSH] OR "Lumbar Vertebrae" $[\mathrm{MeSH}])$ AND "Spinal Fusion"[MeSH]) OR "lumbar fusion"[All Fields] OR ("lumbar"[title] AND "fusion"[title])) AND ("Spondylolisthesis"[MeSH] OR spondylolisthesis[title]) AND (“"2003”[PDAT]: “3000”[PDAT]) AND "humans" [MeSH Terms] AND English[lang]). A total of 134 references were identified. The titles and abstracts of these 134 references were reviewed. Duplicates were discarded, as were nonsystematic reviews, case series, and retrospective cohort studies with fewer than 100 patients. Studies focused on nuances of technique (i.e., choice of bone graft material for fusion) without comparison with nonoperated or nonfused patients were discarded. Studies comparing substantially different procedures (i.e., interbody vs posterolateral fusion) were included in the literature review. Non-English language references were included if there was sufficient translation of key portions of the reference to allow review. The reference lists of previously published systematic reviews were also reviewed to confirm completeness of the literature search. This strategy resulted in 26 primary references and 5 systematic reviews. ${ }^{1-25,27-32}$ Ten papers published since the previous review and one paper that was missed in the previous review providing Level III evidence or better are detailed in the evidentiary table (Table 1).

\section{Scientific Foundation}

\section{Surgery Versus No Surgery}

Weinstein et al.,29,30 through publication of the Spine Patient Outcomes Research Trial (SPORT) studies, provide the most powerful evidence supporting the role of surgical intervention in patients with stenosis associated with degenerative spondylolisthesis. This large $(>600$ patient) multicenter prospective study was originally designed as a randomized trial, but flaws in the study design and the substantial crossover rate between treatment cohorts have led most, including the authors of this study, to focus on the results of the as-treated analysis. As a result, the randomization process was abandoned and the study regarded as a large well-controlled prospective cohort study. The SPORT group demonstrated that when patients are able to select their treatment strategy based on their symptoms, values, and surgical recommendation, those who choose surgery experience superior outcomes in every clinical measure and at every time point for at least 4 years following treatment. It is important to note that surgeons treated patients with decompression and fusion and were free to offer patients whatever technique of decompression and fusion they thought appropriate. ${ }^{29,30}$ As a result of the study limitations, the SPORT provides Level II evidence in support of decompression and fusion for stenosis associated with a spondylolisthesis.

In a companion study, Pearson and the SPORT investigators reviewed preoperative radiographic measurements and 1-year follow-up data in an attempt to identify prognostic indicators of outcome following operative or nonoperative management. ${ }^{24}$ Patients in the surgical cohort exhibited superior outcomes compared with those treated nonoperatively; however, there were no preoperative radiographic features that predicted ultimate success. This finding was confounded by the fact that the choice of fusion technique was left to the discretion of the treating surgeons. In the nonoperative arm, better outcomes were paradoxically associated with increased mobility at the level of the listhesis. Confounding factors between the "stable" and "hypermobile" groups such as sex, work status, and compensation status make it difficult to interpret these results. The strength of this study is reduced to Level III evidence supporting the role of surgery for stenosis associated with spondylolisthesis. ${ }^{24}$

\section{Surgical Technique}

Abdu et al. ${ }^{1}$ reviewed the results from the SPORT lumbar spondylolisthesis study and compared results across fusion techniques. The beneficial effects of surgery were maintained over 4 years, and patients reported significant improvement in every primary outcome measure (Oswestry Disability Index [ODI], 36-Item Short Form Health Survey, and visual analog scale [VAS]) compared with their baseline status. No differences in outcome were detected between the different fusion cohorts (noninstrumented PLF, instrumented PLF, and a $360^{\circ}$ approach, instrumented PLF with an interbody graft). The potential for bias exist, however, because surgeons were free to choose the fusion technique, there were impor- 
D. K. Resnick et al.

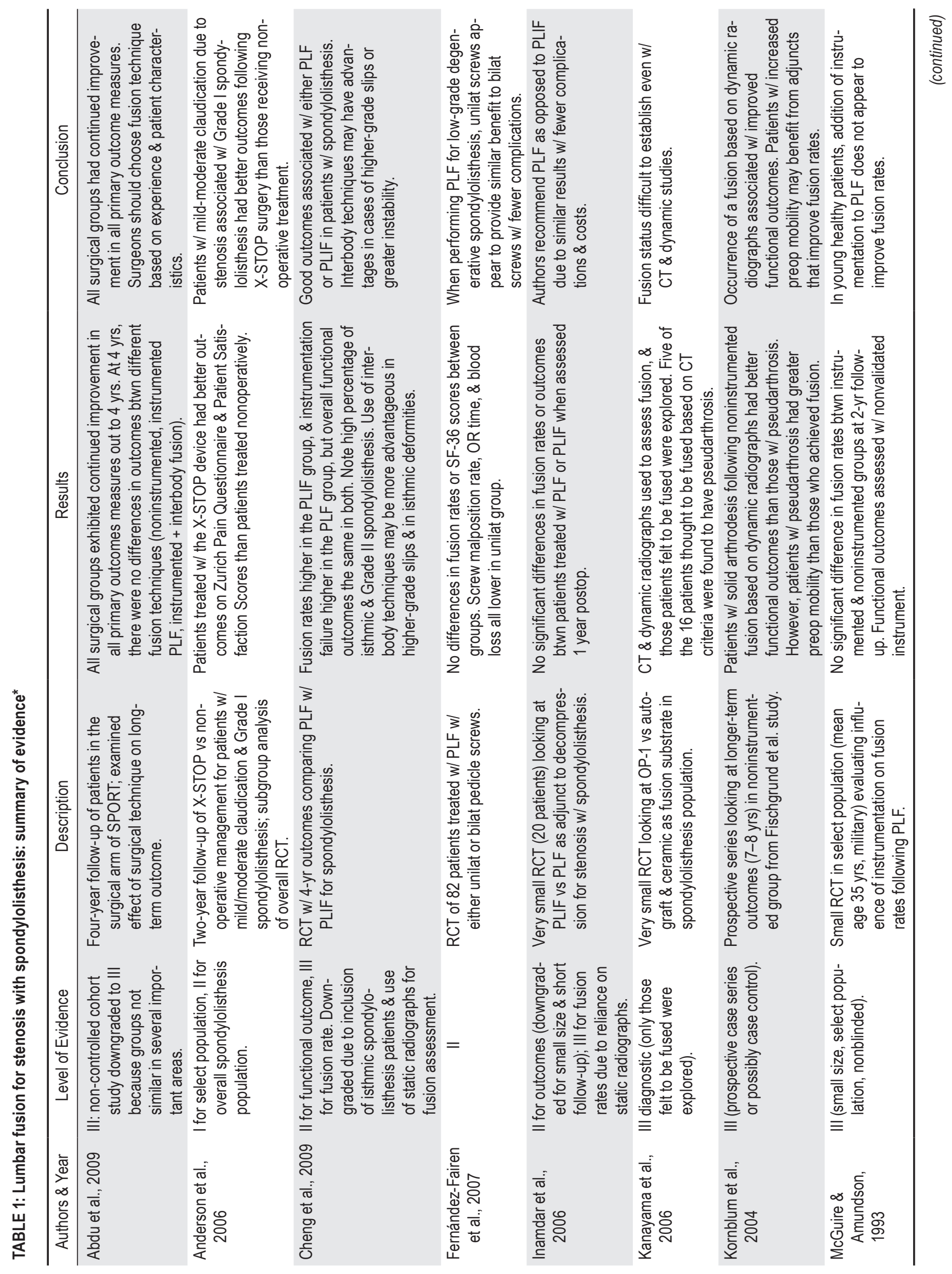


Part 9: Lumbar fusion for stenosis with spondylolisthesis

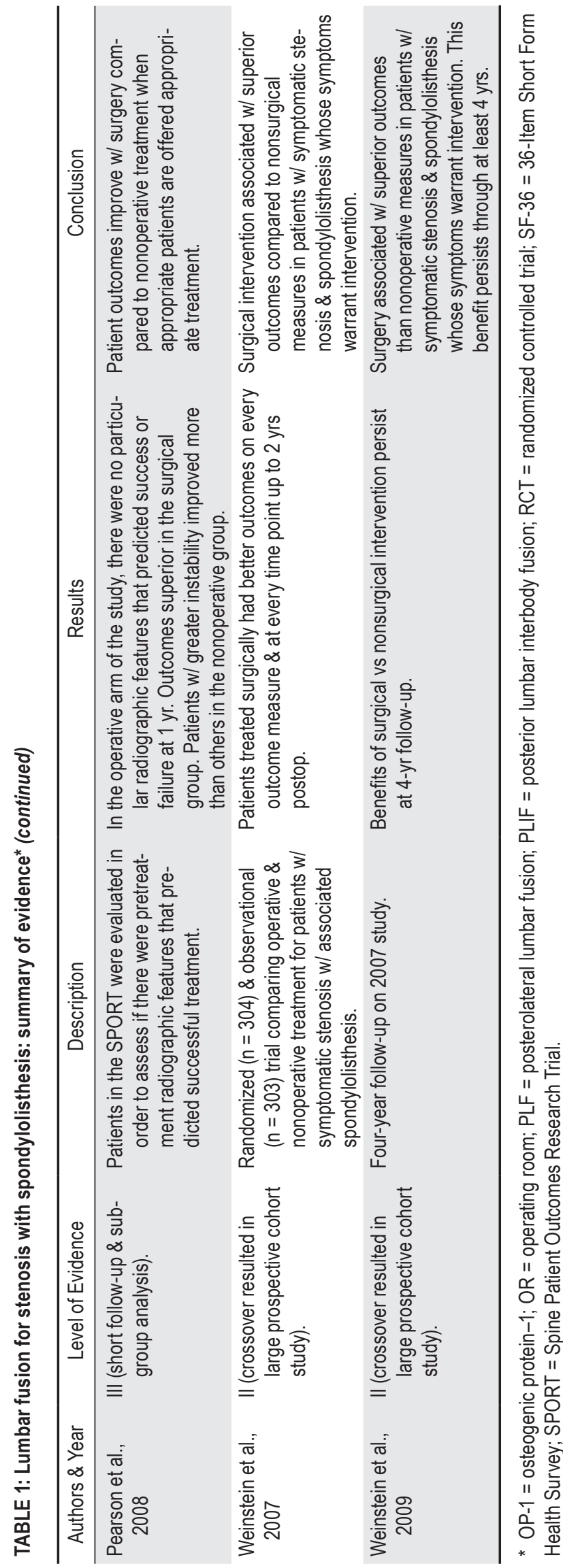

tant demographic differences between the fusion groups (age and race for example), and there were potential differences not described (such as the degree of disc space collapse or regional kyphosis). These confounding factors limit the ability to formulate relevant conclusions regarding the equivalence or nonequivalence of the various fusion techniques. ${ }^{1}$

Cheng and colleagues ${ }^{9}$ performed a randomized trial to evaluate the differences between PLF and posterior lumbar interbody fusion (PLIF) following decompression in a group of 138 patients with degenerative or isthmic spondylolisthesis (Grade I or II). They found that fusion rates were higher and instrumentation-related complication rates were lower in the PLIF group. However, functional outcomes were identical between the groups, and the study relied on static radiographs for the assessment of fusion. The fact that the majority of patients had isthmic spondylolisthesis and that a high percentage of patients had Grade II slips decreases the generalizability of these data to the degenerative population. Due to the heterogeneous patient population and questionable criteria to assess fusion status, the study was downgraded to Level II evidence in support of a PLF or PLIF following decompression for the treatment of degenerative spondylolisthesis. Consideration of interbody techniques may be appropriate in patients with higher-grade slips. ${ }^{9}$

Fernández-Fairen and colleagues ${ }^{12}$ performed a randomized trial in a cohort of 82 patients in whom they examined the effect of unilateral versus bilateral screw fixation as an adjunct to PLF following decompression for degenerative spondylolisthesis. While the sample size was relatively small, the study was powered to detect significant differences on validated outcomes measures and CT scanning was used to determine fusion status 3 years after surgery. The authors group observed no differences in functional outcomes or in fusion rates between the 2 groups and found that complication rates, blood loss, and operative time were lower in the group in which unilateral screws were placed. This study provides Level II evidence that unilateral screw fixation is associated with similar outcomes as bilateral screw fixation, but because the data are generated from a single study with a relatively small patient population, the validity of this conclusion is limited.

Inamdar et al. ${ }^{16}$ performed a randomized study involving 20 patients to investigate the differences in outcomes between PLF and PLIF following decompression for stenosis associated with spondylolisthesis. Clinical and radiographic follow-up data were limited to 1 year. Fusion status was assessed using static radiographs. Although no differences were detected between the treatment groups, the small sample size, short follow-up duration, and questionable method of fusion assessment compromise the conclusions formulated by the authors; therefore, this study is downgraded to Level II evidence in support of PLF over PLIF (Level II for outcomes and Level III for fusion status). ${ }^{16}$

Kornblum and colleagues ${ }^{19}$ followed up the noninstrumented cohort from the Fischgrund et al. study ${ }^{13}$ for a mean of 7.7 years. They followed up 47 of the original 58 patients: only 1 patient was lost to follow-up, 8 died, 1 


\section{K. Resnick et al.}

was disabled from a stroke, and 1 declined to participate. They found that patients in this group who were thought to have a solid arthrodesis (based on dynamic radiographs) enjoyed better functional outcomes (as measured using VAS for pain assessment and the Stucki inventory) than patients treated with the same procedure in whom a solid arthrodesis was not achieved. ${ }^{13,19}$ It was noted that those patients in whom arthrodesis was not achieved had significantly greater preoperative angular mobility. This paper provides Level III evidence as a case-control study showing that efforts to increase fusion rates are associated with better outcomes in patients treated with fusion as an adjunct to decompression.

McGuire and Amundson ${ }^{20}$ studied a military population of patients with stenosis and spondylolisthesis and randomized a total of 27 patients to decompression and fusion with or without instrumentation. Fusion rates at 2 years, based on assessment of flexion-extension radiographs, were similar between the groups (72\% without instrumentation vs $78 \%$ with instrumentation). This paper is felt to provide Level III evidence (small study, nonblinded, very select population with mean age of 35 years) that the addition of instrumentation does not improve fusion rates. ${ }^{20}$ This paper was not included in the previous systematic review. ${ }^{25}$

Other papers have been discussed previously or provide lower-quality evidence. Since some of these provided the basis for the past recommendations, they are briefly discussed below.

Andersen et al. ${ }^{2}$ described long-term outcomes following instrumented and noninstrumented fusion for chronic low-back pain but did not separate out patients with degenerative lumbar spondylolisthesis. This is the same patient cohort previously described by Bjarke Christensen et al. ${ }^{6}$

Athiviraham and Yen $^{5}$ described a cohort series of patients treated nonoperatively, with decompression alone, or with decompression and fusion. Only patients with spondylolisthesis underwent fusion. Due to this important difference between the patient groups in this prospective comparison, this paper is felt to provide only Level IV evidence.

Bridwell and colleagues ${ }^{7}$ performed a pseudo-randomized study involving 43 patients treated operatively for stenosis associated with spondylolisthesis. Nine patients underwent decompression alone; 10, decompression and noninstrumented PLF; and 24, decompression and instrumented PLF. Functional outcomes were better in the fusion group, and better functional outcomes were associated with arrest of slip progression and solid fusion. The use of instrumentation appeared to improve fusion rates as well as patient outcomes. The study was downgraded to a Level III study because the investigators used nonvalidated outcomes measures and relied on static radiographs for the determination of fusion. ${ }^{7}$ This paper was previously reviewed in the 2005 Fusion Guidelines. ${ }^{25}$

Carreon and colleagues ${ }^{8}$ performed a systemic review of the literature to evaluate the effects of fusion on different patient populations. They found that the presence of an established diagnosis such as spondylolisthesis was associated with better functional outcomes compared with patients treated with similar procedures for chronic low-back pain without a demonstrable deformity. Because the analysis included very few spondylolisthesis patients (96 of 2002) and because the index studies are discussed elsewhere in this Guideline Update, the Carreon et al. review does not provide unique information regarding the treatment of this patient population. It does provide supporting evidence confirming that good outcomes may be expected in patients treated with fusion for degenerative spondylolisthesis.

Chou et al. ${ }^{10}$ performed a systematic review of the literature regarding the surgical versus nonsurgical management of low-back pain. While fusion for patients with stenosis was evaluated, spondylolisthesis and nonspondylolisthesis groups were considered together. No specific information regarding the treatment of patients with stenosis and associated spondylolisthesis is given.

Christensen and colleagues ${ }^{11}$ randomized 130 patients with isthmic spondylolisthesis, primary degenerative instability (back pain associated with movement and degenerative disc disease), or secondary degenerative instability (same as primary but with history of having undergone decompression) to PLF with or without instrumentation. No differences between the 2 groups were detected; however, the patient population is not relevant to a discussion of patients with stenosis and degenerative spondylolisthesis. Andersen et al. ${ }^{2}$ described long-term outcomes following instrumented and noninstrumented fusion for chronic low-back pain but did not separate out patients with degenerative lumbar spondylolisthesis. This is the same patient cohort previously described by Bjarke Christensen et al. ${ }^{6}$

Fischgrund and colleagues ${ }^{13}$ performed a prospective clinical trial of 68 patients with stenosis and degenerative spondylolisthesis who were randomized into one of 2 groups: decompression and PLF in one group and decompression and PLF supplemented with pedicle screw fixation in the other. Fusion status was assessed using plain and dynamic radiography, and clinical outcomes were assessed using a VAS for pain as well as a patient satisfaction scale. The patients treated with pedicle screw fixation had a statistically significantly higher fusion rate $(83 \%)$ than those treated with noninstrumented fusion $(45 \%)$. Both groups demonstrated significant score improvements on the VAS for both back and leg pain ( $\mathrm{p}=$ 0.001 ), and the majority of patients in both groups reported their outcomes as good or excellent (78\% in the instrumented group and $85 \%$ in the noninstrumented group). This paper provides Level I medical evidence that pedicle screw fixation, as an adjunct to decompression and PLF, improves fusion success, and Level III medical evidence (due to the nonvalidated patient satisfaction scale and inadequate sample size), suggesting that pedicle screw fixation does not improve functional outcome following PLF in this patient population. ${ }^{13}$ This paper was previously discussed in the 2005 Fusion Guidelines. ${ }^{25}$

Gibson and Waddell ${ }^{14}$ performed a systematic review of randomized trials for the Cochrane Review in 2005. The authors did not review any references not reviewed in the previous guidelines document and did not consider patients with stenosis and spondylolisthesis separately. ${ }^{25}$ 
Kanayama and colleagues ${ }^{17}$ performed a small randomized controlled trial comparing osteogenic protein-1 (OP-1) to autograft plus ceramic as fusion materials in a group of 19 patients undergoing instrumented PLF following decompression for stenosis associated with spondylolisthesis. The OP-1 group was found to have a slightly lower fusion rate as judged by CT scans, dynamic radiographs, and exploration. While new bone formation was noted in both groups, patients who underwent surgical reexploration for planned instrumentation removal were found to have a relatively high incidence of nonunion despite CT- and dynamic radiography-documented evidence of fusion. This paper does not contribute much to the discussion of treatment options for patients with stenosis and spondylolisthesis but does provide information regarding the limitations of imaging studies to provide information regarding the presence or absence of fusion (Level III diagnostic study as patients without radiographic fusion were not surgically explored to confirm/ refute fusion status).

Kondrashov and colleagues ${ }^{18}$ followed up 18 patients treated with the X-STOP device and found that beneficial effects appeared to be durable for a mean of 4.2 years of follow-up in their series (Level IV evidence).

McNeely et al. ${ }^{21}$ performed a systematic review of the effect of physiotherapy on back pain in patients with various diagnoses including spondylolisthesis. They found that there was a paucity of evidence to support the effectiveness of physiotherapy for patients with degenerative spondylolisthesis. This paucity is the result of very few studies and the fact that patients with degenerative spondylolisthesis were not necessarily considered separately. Two randomized studies were reviewed: one on younger patients with isthmic spondylolisthesis ${ }^{23}$ and the other on patients with chronic low-back pain and a variety of spinal alignments but without claudication. ${ }^{27}$

Mirza and Deyo ${ }^{22}$ performed a systematic review of trials evaluating the surgical management of low-back pain. The review did not separately consider patients with stenosis and spondylolisthesis.

Thomsen et al. ${ }^{28}$ performed a randomized controlled clinical trial of 130 patients who underwent lumbar fusion for low-back pain. The patients were randomized to instrumented (pedicle screw fixation) and noninstrumented PLF groups. Overall, there was no significant difference in functional outcome (as measured by the Dallas Pain Questionnaire). Although this paper describes a randomized controlled trial with validated outcome measures, the overall patient population was not that of stenosis and associated spondylolisthesis (isthmic spondylolisthesis, primary and secondary degenerative instability). Only a small subgroup of patients underwent decompression, and it is unclear whether these patients had associated spondylolisthesis. This paper was previously reviewed in the 2005 Fusion Guidelines.

Welch et al. ${ }^{31}$ provided information regarding a prospective case series of patients with stenosis and degenerative spondylolisthesis who were treated with a dynamic fixation device. Overall results appeared promising; however, no comparison cohort was described. This paper is felt to provide Level IV information regarding the poten- tial utility of dynamic fixation in select patients with stenosis and degenerative spondylolisthesis. ${ }^{31}$

Zucherman et al. ${ }^{32}$ performed a prospective randomized study to assess the efficacy of the X-STOP device for the treatment of mild to moderate neurogenic claudication. The results relevant to this discussion have been presented by Anderson et al. ${ }^{3}$ and discussed previously.

\section{Summary}

The current medical evidence continues to support the role of surgery over nonoperative therapies for patients with symptomatic stenosis associated with spondylolisthesis. The vast majority of patients across these studies underwent an instrumented PLF. The achievement of a solid arthrodesis is associated with superior outcomes, and therefore, efforts to maximize fusion potential should be considered. A variety of surgical alternatives may be considered. Surgeons should choose the technique based on their own experience, the risk of complications, and the individual patient's anatomical and physiological characteristics, comorbidities, and preference. It is recognized, however, that within this patient population significant heterogeneity exists that may have an impact on treatment response.

\section{Key Issues for Future Investigation}

The utility of surgical intervention in this patient population is well established. Future work should focus on identifying prognostic indicators of surgical outcome and stratify these factors among the various fusion techniques. Establishing well-designed randomized control trials to address these issues will be extremely difficult if not impractical (as exemplified by the SPORT), but relevant data may be obtained by establishing a prospective diagnosis-based registry.

\section{Acknowledgments}

We would like to acknowledge the AANS/CNS Joint Guidelines Committee (JGC) for their review, comments, and suggestions; Laura Mitchell, CNS Guidelines Project Manager, for her organizational assistance; and Linda O'Dwyer, medical librarian, for assistance with the literature searches. We would also like to acknowledge the following individual JGC members for their contributions throughout the review process: Timothy Ryken, M.D.; Kevin Cockroft, M.D.; Sepideh Amin-Hanjani, M.D.; Steven N. Kalkanis, M.D.; John O’Toole, M.D., M.S.; Steven Casha, M.D., Ph.D.; Aaron Filler, M.D., Ph.D., F.R.C.S.; Daniel Hoh, M.D.; Steven Hwang, M.D.; Todd McCall, M.D.; Jeffrey J. Olson, M.D.; Julie Pilitsis, M.D., Ph.D.; Joshua Rosenow, M.D.; and Christopher Winfree, M.D.

\section{Disclosure}

Administrative costs of this project were funded by the Congress of Neurological Surgeons and the Joint Section on Disorders of the Spine and Peripheral Nerves of the American Association of Neurological Surgeons and Congress of Neurological Surgeons. No author received payment or honorarium for time devoted to this project. Dr. Ghogawala receives grants from the Patient Centered Outcomes Research Institute (PCORI) and the National Institutes of Health (NIH). Dr. Groff is a consultant for DePuy Spine and EBI 
Spine. Dr. Mummaneni owns stock in Spinicity and receives honoraria from DePuy Spine and Globus and royalties from DePuy Spine, Quality Medical Publishers, and Thieme Publishing. Dr. Wang owns stock in Bone Biologics, AxioMed, Amedica, CoreSpine, Expanding Orthopedics, Pioneer, Syndicom, VG Innovations, PearlDiver, Flexuspine, Axis, FzioMed, Benvenue, Promethean, Nexgen, ElectroCore, and Surgitech and holds patents with and receives royalties from Biomet, Stryker, SeaSpine, Aesculap, Osprey, Amedica, Synthes, and Alphatec. The authors report no other potential conflicts of interest concerning the materials or methods used in this study or the findings specified in this paper.

Author contributions to the study and manuscript preparation include the following. Acquisition of data: all authors. Analysis and interpretation of data: all authors. Drafting the article: Resnick. Critically revising the article: all authors. Reviewed submitted version of manuscript: all authors. Approved the final version of the manuscript on behalf of all authors: Resnick. Study supervision: Kaiser.

\section{References}

1. Abdu WA, Lurie JD, Spratt KF, Tosteson AN, Zhao W, Tosteson TD, et al: Degenerative spondylolisthesis: does fusion method influence outcome? Four-year results of the spine patient outcomes research trial. Spine (Phila Pa 1976) 34:23512360, 2009

2. Andersen T, Videbaek TS, Hansen ES, Bünger C, Christensen FB: The positive effect of posterolateral lumbar spinal fusion is preserved at long-term follow-up: a RCT with 11-13 year follow-up. Eur Spine J 17:272-280, 2008

3. Anderson PA, Tribus CB, Kitchel SH: Treatment of neurogenic claudication by interspinous decompression: application of the X STOP device in patients with lumbar degenerative spondylolisthesis. J Neurosurg Spine 4:463-471, 2006

4. Arts MP, Verstegen MJ, Brand R, Koes BW, van den Akker ME, Peul WC: Cost-effectiveness of decompression according to Gill versus instrumented spondylodesis in the treatment of sciatica due to low grade spondylolytic spondylolisthesis: a prospective randomised controlled trial [NTR1300]. BMC Musculoskelet Disord 9:128, 2008

5. Athiviraham A, Yen D: Is spinal stenosis better treated surgically or nonsurgically? Clin Orthop Relat Res 458:90-93, 2007

6. Bjarke Christensen F, Stender Hansen E, Laursen M, Thomsen K, Bünger CE: Long-term functional outcome of pedicle screw instrumentation as a support for posterolateral spinal fusion: randomized clinical study with a 5-year follow-up. Spine (Phila Pa 1976) 27:1269-1277, 2002

7. Bridwell KH, Sedgewick TA, O'Brien MF, Lenke LG, Baldus $\mathrm{C}$ : The role of fusion and instrumentation in the treatment of degenerative spondylolisthesis with spinal stenosis. J Spinal Disord 6:461-472, 1993

8. Carreon LY, Glassman SD, Howard J: Fusion and nonsurgical treatment for symptomatic lumbar degenerative disease: a systematic review of Oswestry Disability Index and MOS Short Form-36 outcomes. Spine J 8:747-755, 2008

9. Cheng L, Nie L, Zhang L: Posterior lumbar interbody fusion versus posterolateral fusion in spondylolisthesis: a prospective controlled study in the Han nationality. Int Orthop 33: 1043-1047, 2009

10. Chou R, Baisden J, Carragee EJ, Resnick DK, Shaffer WO, Loeser JD: Surgery for low back pain: a review of the evidence for an American Pain Society Clinical Practice Guideline. Spine (Phila Pa 1976) 34:1094-1109, 2009

11. Christensen FB, Thomsen K, Eiskjaer SP, Hansen ES, Fruensgaard S, Gelinick J, et al: [The effect of pedicle screw instrumentation on posterolateral spinal fusion. A prospective, randomized study with a two-year follow-up.] Ugeskr Laeger 161:1920-1925, 1999 (Danish)

12. Fernández-Fairen M, Sala P, Ramírez H, Gil J: A prospective randomized study of unilateral versus bilateral instrumented posterolateral lumbar fusion in degenerative spondylolisthesis. Spine (Phila Pa 1976) 32:395-401, 2007

13. Fischgrund JS, Mackay M, Herkowitz HN, Brower R, Montgomery DM, Kurz LT: 1997 Volvo Award Winner in Clinical Studies. Degenerative lumbar spondylolisthesis with spinal stenosis: a prospective, randomized study comparing decompressive laminectomy and arthrodesis with and without spinal instrumentation. Spine (Phila Pa 1976) 22:2807-2812, 1997

14. Gibson JN, Waddell G: Surgery for degenerative lumbar spondylosis: updated Cochrane Review. Spine (Phila Pa 1976) 30: 2312-2320, 2005

15. Grob D, Humke T, Dvorak J: [Significance of simultaneous fusion and surgical decompression in lumbar spinal stenosis.] Orthopade 22:243-249, 1993 (Ger)

16. Inamdar DN, Alagappan M, Shyam L, Devadoss S, Devadoss A: Posterior lumbar interbody fusion versus intertransverse fusion in the treatment of lumbar spondylolisthesis. J Orthop Surg (Hong Kong) 14:21-26, 2006

17. Kanayama M, Hashimoto T, Shigenobu K, Yamane S, Bauer TW, Togawa D: A prospective randomized study of posterolateral lumbar fusion using osteogenic protein-1 (OP-1) versus local autograft with ceramic bone substitute: emphasis of surgical exploration and histologic assessment. Spine (Phila Pa 1976) 31:1067-1074, 2006

18. Kondrashov DG, Hannibal M, Hsu KY, Zucherman JF: Interspinous process decompression with the X-STOP device for lumbar spinal stenosis: a 4-year follow-up study. J Spinal Disord Tech 19:323-327, 2006

19. Kornblum MB, Fischgrund JS, Herkowitz HN, Abraham DA, Berkower DL, Ditkoff JS: Degenerative lumbar spondylolisthesis with spinal stenosis: a prospective long-term study comparing fusion and pseudarthrosis. Spine (Phila Pa 1976) 29:726-734, 2004

20. McGuire RA, Amundson GM: The use of primary internal fixation in spondylolisthesis. Spine (Phila Pa 1976) 18:16621672,1993

21. McNeely ML, Torrance G, Magee DJ: A systematic review of physiotherapy for spondylolysis and spondylolisthesis. Man Ther 8:80-91, 2003

22. Mirza SK, Deyo RA: Systematic review of randomized trials comparing lumbar fusion surgery to nonoperative care for treatment of chronic back pain. Spine (Phila Pa 1976) 32:816823,2007

23. O'Sullivan PB, Phyty GD, Twomey LT, Allison GT: Evaluation of specific stabilizing exercise in the treatment of chronic low back pain with radiologic diagnosis of spondylolysis or spondylolisthesis. Spine (Phila Pa 1976) 22:2959-2967, 1997

24. Pearson AM, Lurie JD, Blood EA, Frymoyer JW, Braeutigam H, An H, et al: Spine Patient Outcomes Research Trial: radiographic predictors of clinical outcomes after operative or nonoperative treatment of degenerative spondylolisthesis. Spine (Phila Pa 1976) 33:2759-2766, 2008

25. Resnick DK, Choudhri TF, Dailey AT, Groff MW, Khoo L, Matz PG, et al: Guidelines for the performance of fusion procedures for degenerative disease of the lumbar spine. Part 9: fusion in patients with stenosis and spondylolisthesis. J Neurosurg Spine 2:679-685, 2005

26. Resnick DK, Choudhri TF, Dailey AT, Groff MW, Khoo L, Matz PG, et al: Guidelines for the performance of fusion procedures for degenerative disease of the lumbar spine. Part 12: pedicle screw fixation as an adjunct to posterolateral fusion for low-back pain. J Neurosurg Spine 2:700-706, 2005

27. Spratt KF, Weinstein JN, Lehmann TR, Woody J, Sayre H: Efficacy of flexion and extension treatments incorporating braces for low-back pain patients with retrodisplacement, spondylolisthesis, or normal sagittal translation. Spine (Phila Pa 1976) 18:1839-1849, 1993

28. Thomsen K, Christensen FB, Eiskjaer SP, Hansen ES, Fruensgaard S, Bünger CE: 1997 Volvo Award Winner in Clini- 


\section{Part 9: Lumbar fusion for stenosis with spondylolisthesis}

cal Studies. The effect of pedicle screw instrumentation on functional outcome and fusion rates in posterolateral lumbar spinal fusion: a prospective, randomized clinical study. Spine (Phila Pa 1976) 22:2813-2822, 1997

29. Weinstein JN, Lurie JD, Tosteson TD, Hanscom B, Tosteson AN, Blood EA, et al: Surgical versus nonsurgical treatment for lumbar degenerative spondylolisthesis. N Engl J Med 356: 2257-2270, 2007

30. Weinstein JN, Lurie JD, Tosteson TD, Zhao W, Blood EA, Tosteson AN, et al: Surgical compared with nonoperative treatment for lumbar degenerative spondylolisthesis. Four-year results in the Spine Patient Outcomes Research Trial (SPORT) randomized and observational cohorts. J Bone Joint Surg Am 91:1295-1304, 2009

31. Welch WC, Cheng BC, Awad TE, Davis R, Maxwell JH, Delamarter R, et al: Clinical outcomes of the Dynesys dynamic neutralization system: 1-year preliminary results. Neurosurg Focus 22(1):E8, 2007
32. Zucherman JF, Hsu KY, Hartjen CA, Mehalic TF, Implicito DA, Martin MJ, et al: A multicenter, prospective, randomized trial evaluating the X STOP interspinous process decompression system for the treatment of neurogenic intermittent claudication: two-year follow-up results. Spine (Phila Pa 1976) 30:1351-1358, 2005

Manuscript submitted March 13, 2014.

Accepted April 3, 2014.

Please include this information when citing this paper: DOI: 10.3171/2014.4.SPINE14274.

Address correspondence to: Michael G. Kaiser, M.D., Columbia University, Neurological Surgery, The Neurological Institute, 710 W. 168th St., New York, NY 10032.email: mgk7@columbia.edu. 